\title{
Polya Counting Theory Applied to Combination of Edge Conditions for Generally Shaped Isotropic Plates
}

\author{
Yoshihiro Narita ${ }^{a *}$ \\ ${ }^{a}$ Hokkaido University (Professor Emeritus), C-BEST Project, Center of Technology, UNHAS. Email: ynarita@eng.hokudai.ac.jp
}

\begin{abstract}
Structural behaviors of plate components, such as internal stress, deflection, buckling and dynamic response, are important in the structural design of aerospace, mechanical, civil and other industries. These behaviors are known to be affected not only by plate shapes and material properties but also by edge conditions. Any one of the three classical edge conditions in bending, namely free, simply supported and clamped edges, may be used to model the constraint along an edge of plates. Along the entre boundary with plural edges, there exist a wide variety of combinations in the entire plate boundary, each giving different values of structural responses. For counting the total number of possible combinations, the present paper considers Polya counting theory in combinatorial mathematics. For various plate shapes, formulas are derived for counting exact numbers in combination. In some examples, such combinations are confirmed in the figures by a trial and error approach.
\end{abstract}

Keywords: Counting problem; combinatory mathematics; plate shape; Polya counting theory; structural response

\section{Introduction}

Many structural panels are found and can be modelled in the form of flat plates in the fields of mechanical, civil, aeronautical, ocean and other industries. Technical publications are therefore numerous on mechanics of flat plates. For example, a monograph "Vibration of Plates" was compiled by Leissa [1] in 1969 to cover the topic from vibrational viewpoint. He also listed up the natural frequencies of isotropic rectangular plates for all the possible combinations of classical edge conditions [2].

Apart from vibrational aspect, it is important to know in design process the mechanical behaviors, such as stress and strain, deflection and critical buckling loads. These values are influenced significantly by edge conditions (The edge conditions are stated as boundary conditions in mathematics, but in the present paper, the terminology "edge condition" is used throughout the paper from mechanics viewpoint), and should be summarized as sets of design data in comprehensive fashion. To the present, however, little efforts have been made to clarify even the number of combinations in terms of edge conditions. For this purpose, an attempt is made in this paper to count all possible combinations of edge conditions. Mathematically, it is interpreted as combinatorics problem of counting different

*Corresponding author.

Kita-13, Nishi-8, Kita-ku, Sapporo, Japan 060-8628 classes (combinations) that give different sets of results on stress and so on, as the values mentioned above.

As for methodology, Polya counting theory is used. Polya made fundamental contributions to combinatorics, number theory, numerical analysis and probability theory. The theory is also known as Polya enumeration theorem and Redfield-Polya theorem. It is a theorem in combinatorics that generalizes Burnside's lemma on the number of orbits of a group action on a set. After the first publication by Redfield in 1927 [3], Polya developed the theory [4] as a powerful tool in combinatorics and graph theory (for example, refer to textbook [5]), and popularized by applying it to many counting problems. Particularly, it has a wide range of applications to enumeration of chemical compounds. For example, Haigh and Baker [6] applied it to enumeration of the isomers in chemistry.

In the present paper, the theory is used to count the number of combinations of edge conditions that yield different mechanical behaviours. Such behaviours include stress distribution, deflection, natural frequencies and critical buckling loads found in isotropic flat plates with general shapes. After the method is outlined for counting the present cases, cyclic polynomials are derived and used to calculate eighteen examples with different edge numbers when the number of each edge condition is given for $1,2,3$ 
and 4. The total number of sets of edge conditions is listed for eighteen different plate shapes. For showing validity of these results, actual combinations are listed for rhombic, symmetric trapezoidal, equilateral and isosceles triangular and regular pentagonal plates in the case of two edge conditions (free edge and clamped edge) by a trial and error approach.

\section{Polya Counting Theory}

The combinatorics approach is outlined here as used in [7]. In Group theory, a permutation is defined as a one-to-one mapping from a set $\boldsymbol{D}$ onto $\boldsymbol{D}$. For example, when there is a set $\boldsymbol{D}$ composed of four elements $\{1,2,3,4\}$, i.e., $\boldsymbol{D}=\{1,2,3,4\}$, a permutation of transposing $1 \rightarrow 2,2 \rightarrow 4,3 \rightarrow 3,4 \rightarrow 1$ can be written as

$$
P=\left(\begin{array}{llll}
1 & 2 & 3 & 4 \\
2 & 4 & 3 & 1
\end{array}\right)
$$

or more simply, a cyclic notation

$$
P=\{(124)(3)\}
$$

can be used as used in this paper. For such two permutations, it is clear that a product $\boldsymbol{P}_{1} \boldsymbol{P}_{2}$ of $\boldsymbol{P}_{1}$ and $\boldsymbol{P}_{2}$ also becomes a permutation. In Group theory, a set $\boldsymbol{G}$ of all permutations acting on a set $\boldsymbol{D}$ can be considered as a finite group. The reason is that the associative law exists with a unit and inverse elements in $\boldsymbol{G}$. In the present problem, a set composed of all possible rotation and flipping of a plate shape becomes such a permutation group.

The next important notation is "class" in counting problem. In this paper, this can be explained next by using a case of square plate. Figure 1 presents a square isotropic plate (i.e, plate made of no material anisotropy), and four edges are numbered as Edge 1 (left-hand edge), Edge 2 (lower edge), Edge 3 (right-hand edge) and Edge 4 (upper edge) in counter-clock wise. When one of classical edge conditions of free (denoted by F), simply-supported (S) and clamped (C) in bending is applied to each of the four edges, the notation of entire edges is given by four capital letters, for example, CSFF is the plate with calmed (C) at Edge 1, simply supported (S) at Edge 2 and free (F) at Edge 3 and 4.

The square plate in Fig.1 now has four edges under three different boundary conditions (F,S,C), and when the four edges are fixed in the space, the number of edge conditions is fixed by $3^{4}=81$. However, when this combination problem is looked from mechanical behaviors such as stress distribution, deflection, vibration frequencies, and critical buckling loads, two cases of CSFF and FCSF are identical because one case FCSF is obtained by rotating CSFF with 90 degree in counter-clockwise. These two cases are said to belong to the same class. In contrast, other cases of CSFF and CFSF (the same number of C,S,F exist) cannot be in the same class, because one case cannot be realized either by rotation or flipping. This paper considers counting the number of classes when a cyclic permutation group $\boldsymbol{G}$ acts on a set $\boldsymbol{D}$.

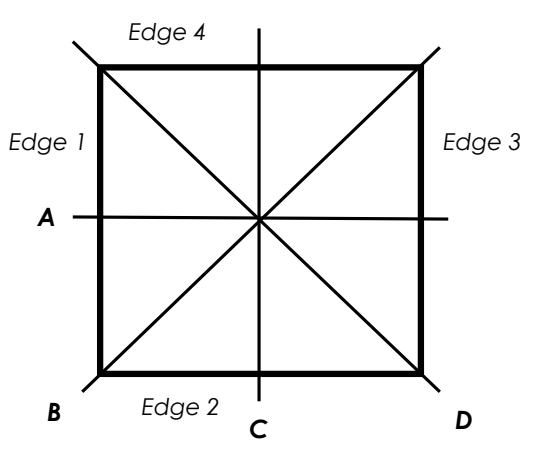

Figure 1. Square plate and symmetry axes (Ex.1)

Polya counting theory $[4,5]$ derives a kind of polynomial "cyclic polynomial" in order to calculate the number of essentially different cases (i.e, number of different "classes"). When a cyclic group, acting on a finite group, is denoted by $\boldsymbol{G}$ and $C_{k}(\boldsymbol{G})$ is a number of elements in $\boldsymbol{G}$ with the cyclic number $k$, the cyclic polynomial for a group $\boldsymbol{G}$ acting on $\boldsymbol{D}$ is given by

$$
Z_{G}(x)=\frac{1}{|G|} \sum_{k=1}^{|G|} C_{k}(G) x^{k}
$$

\section{Application of the Theory and Cyclic Polynomials}

\subsection{Plates with four straight edges}

\subsubsection{Square plate (Ex.1)}

As shown in Fig.1, a square plate has four edges of equal length and four symmetrical axes. Two axes are parallel to the edges (labeled as $\boldsymbol{A}$ and $\boldsymbol{C}$ in the figure), and remaining two axes are diagonal axes labelled as $\boldsymbol{B}$ and $\boldsymbol{D}$.

For rotation around the central point, it has four possible rotational motions in counter-clockwise as

$$
G_{r}=\{(1)(2)(3)(4),(1234),(13)(24),(1432)\}
$$

with rotating angles $0^{\circ}, 90^{\circ}, 180^{\circ}$ and $270^{\circ}$, respectively.

For flipping, it has four possible flipping motions as

$$
G_{f}=\{(1)(3)(24),(12)(34),(2)(4)(13),(14)(23)\}
$$

by flipping with respect to the symmetric axes $\boldsymbol{A}, \boldsymbol{B}, \boldsymbol{C}$ and $D$, respectively. By adding two sets of motions, one gets

$$
G=G_{r}+G_{f},|G|=8
$$

and number of elements in Eqs.(4) and (5) are given for each cyclic number $k$ as

$$
C_{4}=1, C_{3}=2, C_{2}=3, C_{1}=2
$$

From (6) and (7), a cyclic polynomial in this example is

$$
Z_{G}(x)=\frac{1}{8}\left(x^{4}+2 x^{3}+3 x^{2}+2 x\right)
$$

This case was already obtained in Ref. [7] written by the present author. 


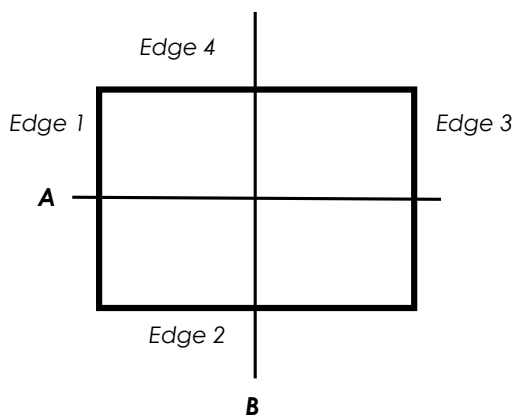

Figure 2. Rectangular plate and symmetry axes (Ex.2)

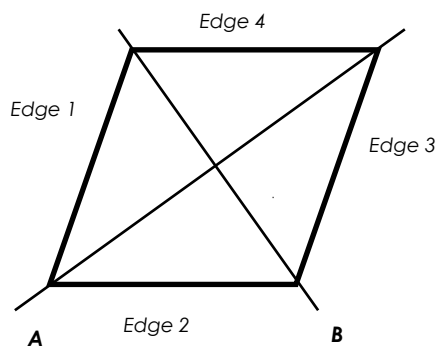

Figure 3. Rhombic plate and symmetry axes (Ex.3)

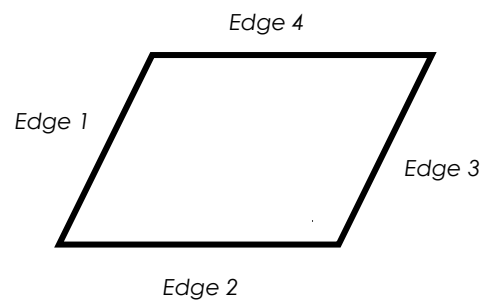

Figure 4. Parallelogram plate (Ex.4)

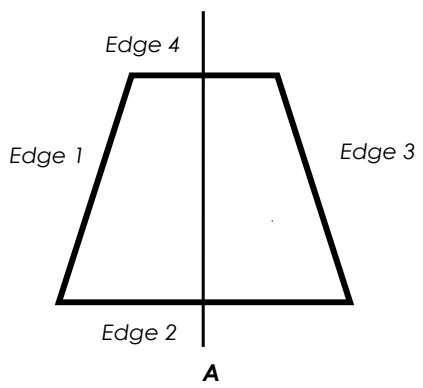

Figure 5. Symmetric trapezoidal plate (Ex.5)

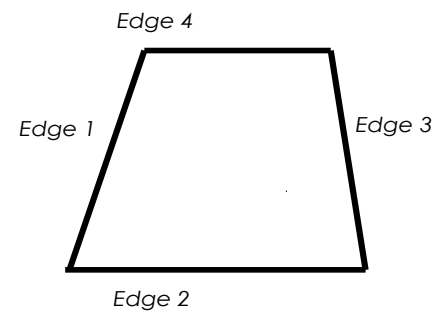

Figure 6. Asymmetric trapezoidal plate (Ex.6)

\subsubsection{Rectangular plate (Ex.2)}

A rectangular plate is generalization of a square plate, and diagonal symmetric axes do not exist, leaving only two symmetric axes parallel to the edges (labelled as $\boldsymbol{A}$ and $\boldsymbol{B}$ ) as shown in Fig. 2. For rotation around the central point, it has four possible rotational motions in counter-clockwise as

$$
G_{r}=\{(1)(2)(3)(4),(13)(24)\}
$$

with angles $0^{\circ}$ and $180^{\circ}$, respectively. It has two flipping motions as

$$
G_{f}=\{(1)(3)(24),(2)(4)(13)\}
$$

by flipping with respect to the symmetric axes $\boldsymbol{A}$ and $\boldsymbol{B}$, respectively. By adding two sets of motions, one gets a number of elements in Eqs.(9) and (10), totally, as

$$
C_{4}=1, C_{3}=2, C_{2}=1, C_{1}=0
$$

A cyclic polynomial in this example is

$$
Z_{G}(x)=\frac{1}{4}\left(x^{4}+2 x^{3}+x^{2}\right)
$$

This case is also obtained in Ref. [7].

\subsubsection{Rhombic plate (Ex.3)}

A rhombus is composed of four edges of equal length, but the corners are no longer perpendicular. There are two symmetric axes connecting opposite corners as shown in Fig.3. For rotation and flipping, the motions are described as motions

$$
G_{r}=\{(1)(2)(3)(4),(13)(24)\}
$$

and

$$
G_{f}=\{(12)(34),(14)(23)\}
$$

by flipping with respect to the symmetric axes $\boldsymbol{A}$ and $\boldsymbol{B}$, respectively. By following the same process, a cyclic polynomial is

$$
Z_{G}(x)=\frac{1}{4}\left(x^{4}+3 x^{2}\right)
$$

\subsubsection{Parallelogram plate (Ex.4)}

As shown in Fig.4, a parallelogram is a geometry where two pairs of parallel straight lines intersect each other. The length of two sets of edges are generally not equal (if they are equal, it becomes a rhombus). There are no symmetry axes in geometry. For rotation and flipping, the motions are

$$
G_{r}=\{(1)(2)(3)(4),(13)(24)\}
$$

and $G_{f}=\{0\}$. A cyclic polynomial is

$$
Z_{G}(x)=\frac{1}{2}\left(x^{4}+x^{2}\right)
$$




\subsubsection{Symmetric trapezoidal plate (Ex.5)}

As shown in Fig. 5, a symmetric trapezoid is a geometry where one pairs of parallel straight lines is intersected by a pair of lines with the same angle. Only one pair of opposite edges is of equal length. There is only one symmetry axis in geometry. For rotation and flipping, the motions are

$$
G_{r}=\{(1)(2)(3)(4)\}, G_{f}=\{(13)(2)(4)\}
$$

A cyclic polynomial is

$$
Z_{G}(x)=\frac{1}{2}\left(x^{4}+x^{3}\right)
$$

\subsubsection{Asymmetric trapezoidal plate (Ex.6)}

An asymmetric trapezoid is a geometry with a pair of two parallel lines but the other two opposite straight lines have different intersecting angles to the parallel lines. This geometry has no symmetric axis as seen in Fig. 6, and has only unit element,

$$
G_{r}=\{(1)(2)(3)(4)\}
$$

and $G_{f}=\{0\}$. A cyclic polynomial is

$$
Z_{G}(x)=x^{4}
$$

\subsection{Triangular Plates}

\subsubsection{Equilateral triangular plate (Ex.7)}

An equilateral triangle is a triangle where all three sides are equal in length, and is also equiangular. All three internal angles are $60^{\circ}$ each. It is also a regular polygon and can be called as a regular triangle. As shown in Fig.7, it has three symmetric axes, labelled as A, B and C. For rotation around the central point, it has three rotational motions in counterclockwise as

$$
G_{r}=\{(1)(2)(3),(123),(132)\}
$$

with $0^{\circ}, 120^{\circ}$ and $240^{\circ}$, respectively. It has two flipping motions as

$$
G_{f}=\{(1)(23),(2)(13),(3)(12)\}
$$

by flipping with respect to the symmetric axes $\boldsymbol{A}, \boldsymbol{B}$ and $\boldsymbol{C}$, respectively. A cyclic polynomial in this example is

$$
Z_{G}(x)=\frac{1}{6}\left(x^{3}+3 x^{2}+2 x\right)
$$

\subsubsection{Isosceles triangular plate (Ex.8)}

An isosceles triangle in Fig. 8 has only one axis of symmetry, and has only one action for each of rotation and flipping. Therefore, a cyclic polynomial is

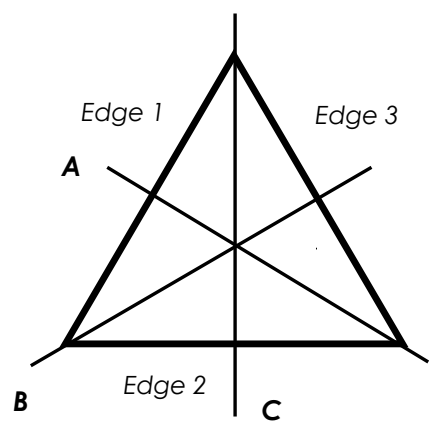

Figure 7. Equilateral triangular plate (Ex.7)

$$
Z_{G}(x)=\frac{1}{2}\left(x^{3}+x^{2}\right)
$$

\subsubsection{Right-angled triangular plate (Ex.9)}

A right-angled triangle has no axis in symmetry, and can be an isosceles triangle when length of two edges is equal. But in this section as shown in Fig.9, all three edges are assumed to be different length, then, there is only an unit action. A cyclic polynomial is

$$
Z_{G}(x)=x^{3}
$$

\subsubsection{General triangular plate (Ex.10)}

For most general case of triangle in Fig.10, there are no edges of equal length. Because it has only unit element,

$$
Z_{G}(x)=x^{3}
$$

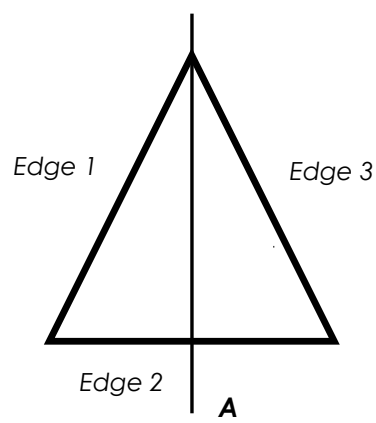

Figure 8. Isosceles triangular plate (Ex.8)

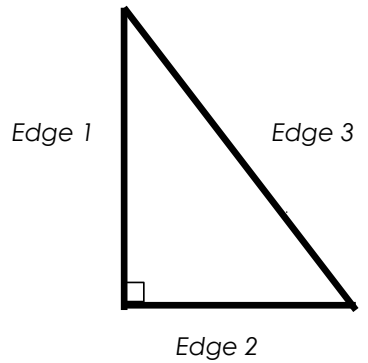

Figure 9. Right-angled triangular plate (Ex.9) 


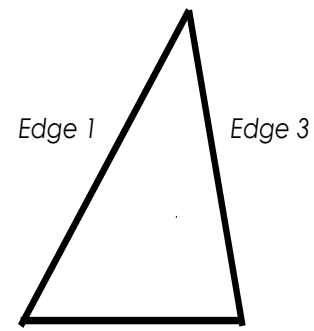

Edge 2

Figure 10. General triangular plate (Ex.10)

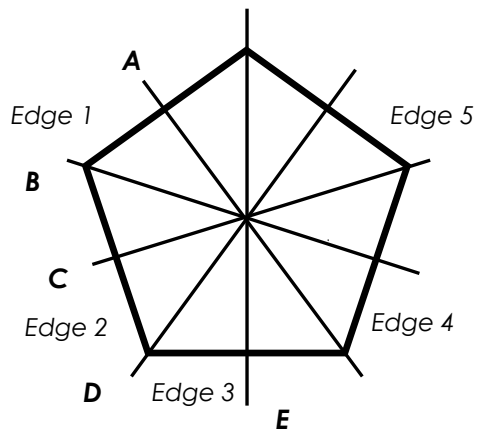

Figure 11. Regular pentagonal plate (Ex.11)

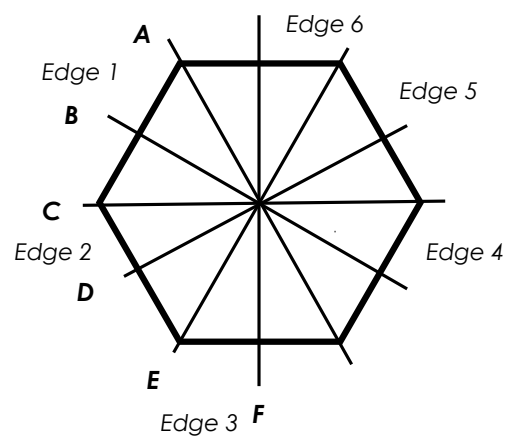

Figure 12. Regular hexagonal plate (Ex.12)

\subsection{Polygonal Plates}

\subsubsection{Regular pentagonal plate (Ex.11)}

A pentagon is a five-sided polygon and the sum of the internal angles is $540^{\circ}$. A self-intersecting regular pentagon is called a pentagram. As shown in Fig. 11, it has five symmetric axes, labelled as $\boldsymbol{A}, \boldsymbol{B}, \boldsymbol{C}, \boldsymbol{D}$ and $\boldsymbol{E}$. For rotation around the central point, it has five rotational motions in counter-clockwise as

$$
\begin{aligned}
G_{r}= & \{(1)(2)(3)(4)(5),(12345),(13524), \\
& (14253),(15432)\}
\end{aligned}
$$

with $0^{\circ}, 72^{\circ}, 144^{\circ}, 216^{\circ}$ and $288^{\circ}$, respectively. It has five flipping motions as

$$
\begin{aligned}
G_{f}= & \{(1)(25)(34),(2)(13)(45),(3)(24)(15), \\
& (4)(12)(35),(5)(23)(14)\}
\end{aligned}
$$

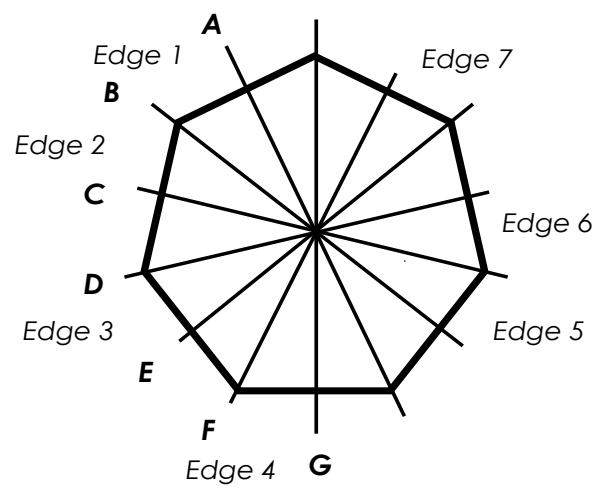

Figure 13. Regular septagonal plate (Ex.13)

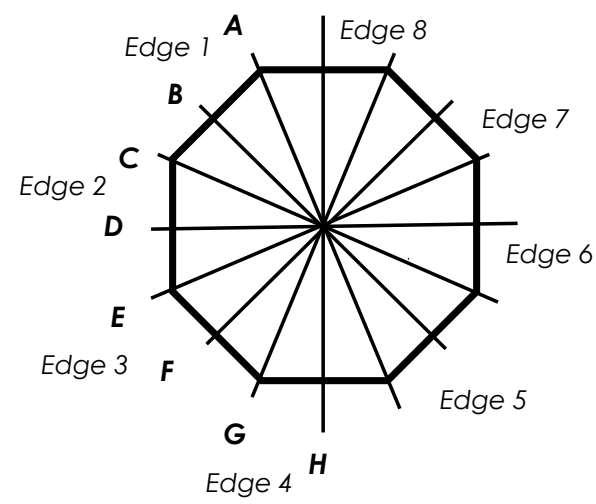

Figure 14. Regular octagonal plate (Ex.14)

by flipping with respect to the symmetric axes $A, B, C, D$ and $\boldsymbol{E}$, respectively. So, a cyclic polynomial in this case is

$$
Z_{G}(x)=\frac{1}{10}\left(x^{5}+5 x^{3}+4 x\right)
$$

\subsubsection{Regular hexagonal plate (Ex.12)}

A regular hexagonal plate has six symmetric axes $(\boldsymbol{A}, \boldsymbol{B}$, $\boldsymbol{C}, \boldsymbol{D}, \boldsymbol{E}$ and $\boldsymbol{F}$ ) as shown in Fig. 12. For rotations,

$$
\begin{aligned}
& G_{r}=\{(1)(2)(3)(4)(5)(6),(123456),(135)(246), \\
& (14)(25)(36),(153)(264),(165432)\}
\end{aligned}
$$

with $0^{\circ}, 60^{\circ}, 120^{\circ}, 180^{\circ}, 240^{\circ}$ and $300^{\circ}$, respectively. It has two flipping motions as

$$
\begin{aligned}
G_{f}= & \{(12)(36)(45),(23)(14)(56), \\
& (34)(25)(16),(3)(6)(24)(15), \\
& (1)(4)(26)(35),(2)(5)(14)(46)\}
\end{aligned}
$$

Then, a cyclic polynomial is

$$
Z_{G}(x)=\frac{1}{12}\left(x^{6}+3 x^{4}+4 x^{3}+2 x^{2}+2 x\right)
$$




\subsubsection{Regular septagonal plate (Ex.13)}

A septagon (or heptagon) is a seven seven-sided polygon, as sown in Fig. 13, it has seven symmetric axes. For rotation around the central point, it has seven rotational motions.

$$
\begin{aligned}
& G_{r}=\{(1)(2)(3)(4)(5)(6)(7),(1234567),(1357246), \\
& (1473625),(1526374),(1642753),(1765432)\}
\end{aligned}
$$

with $0^{\circ}, \alpha, 2 \alpha, 3 \alpha, 4 \alpha, 5 \alpha$ and $6 \alpha\left(\alpha=360 / 7^{\circ}\right)$, respectively. It has seven flipping motions as

$$
\begin{aligned}
& G_{f}=\{(1)(27)(36)(45),(2)(13)(47)(56), \\
& (3)(24)(15)(67),(4)(35)(26)(17), \\
& (5)(46)(37)(12),(6)(14)(23)(57) \\
& (7)(16)(25)(34)\}
\end{aligned}
$$

Therefore, a cyclic polynomial in this case is

$$
Z_{G}(x)=\frac{1}{14}\left(x^{7}+7 x^{4}+6 x\right)
$$

\subsubsection{Regular octagonal plate (Ex.14)}

An octagon is an eight-sided polygon, as sown in Fig. 14, it has eight symmetric axes. For rotation around the central point, it has eight rotational motions.

$$
\begin{aligned}
& G_{r}=\{(1)(2)(3)(4)(5)(6)(7)(8),(12345678), \\
& ,(1357)(2468),(14725836),(15)(26)(37)(48), \quad(37) \\
& (16385274),(1753)(2864),(18765432)\}
\end{aligned}
$$

with $0^{\circ}, 45^{\circ}, 90^{\circ}, 135^{\circ}, 180^{\circ}, 225^{\circ}, 270^{\circ}$ and $315^{\circ}$, respectively. It has seven flipping motions as

$$
\begin{aligned}
& G_{f}=\{(1)(5)(28)(37)(46),(2)(6)(13)(48)(57), \\
& (3)(7)(24)(15)(68),(4)(8)(35)(26)(17), \\
& (12)(38)(47)(56),(23)(14)(58)(67), \\
& (34)(25)(16)(78),(18)(27)(36)(45)\}
\end{aligned}
$$
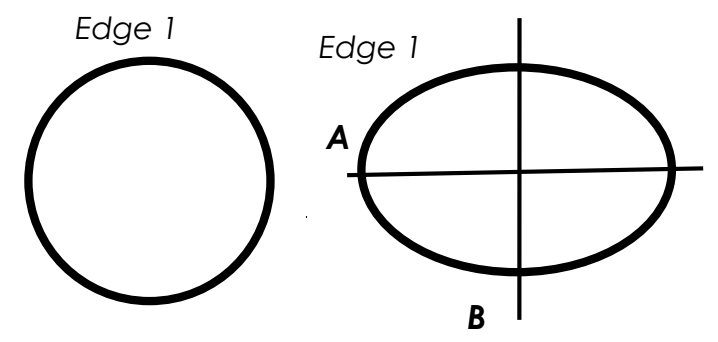

Figure 15. Circular and Elliptical plate (Ex.15 \& 16)
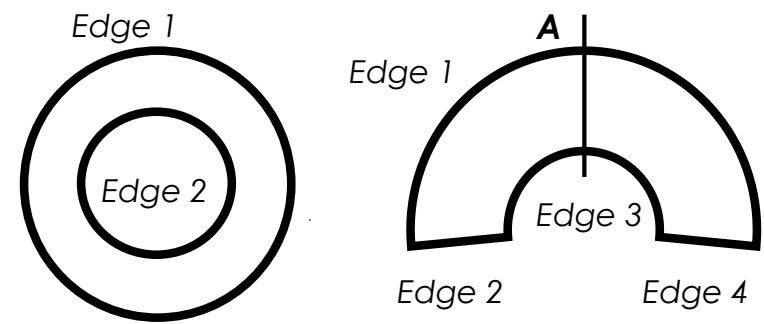

Figure 16. Annular and Sectorial plate (Ex.17 \& 18)

Therefore, a cyclic polynomial in this case is

$$
Z_{G}(x)=\frac{1}{16}\left(x^{8}+4 x^{5}+5 x^{4}+2 x^{2}+4 x\right)
$$

\subsection{Plates with Circular Edges}

\subsubsection{Solid circular plate (Ex.15)}

Except for cases considering mixed edge conditions, circular plate in Fig. 15 has only one edge condition, and so

$$
Z_{G}(x)=x
$$

\subsubsection{Solid elliptical plate (Ex.16)}

Again, except for cases with mixed edge conditions, solid elliptical plate in Fig. 15 has only one edge, and so

$$
Z_{G}(x)=x
$$

\subsubsection{Annular circular plate (Ex.17)}

An annular circular plate in Fig.16 has two edges, and

$$
Z_{G}(x)=x^{2}
$$

\subsubsection{Annular circular sectorial plate (Ex.18)}

As shown in Fig.16, the present plate has two circular and two straight edges, and one axis of symmetry. There is no rotation but one flipping with the axis, i.e.,

$$
G_{r}=\{(1)(2)(3)(4)\}
$$

and

$$
G_{f}=\{(1)(3)(24)\}
$$

Therefore, a cyclic polynomial is

$$
Z_{G}(x)=\frac{1}{2}\left(x^{4}+x^{3}\right)
$$


Table 1. Different classes (combinations) of plates (Ex.1-18) versus number of edge conditions applied along each edge

\begin{tabular}{|c|c|c|c|c|}
\hline & \multicolumn{5}{|c|}{ Number of conditions $\boldsymbol{x}$ per edge } \\
\hline \multicolumn{5}{|c|}{ Plates with four straight edges } \\
\hline Ex.1 & 1 & 6 & 21 & 55 \\
\hline Ex.2 & 1 & 9 & 36 & 100 \\
\hline Ex.3 & 1 & 7 & 27 & 76 \\
\hline Ex.4 & 1 & 10 & 45 & 136 \\
\hline Ex.5 & 1 & 12 & 54 & 160 \\
\hline Ex.6 & 1 & 16 & 81 & 256 \\
\hline \multicolumn{5}{|c|}{ Triangular plates } \\
\hline Ex.7 & 1 & 4 & 10 & 20 \\
\hline Ex.8 & 1 & 6 & 18 & 40 \\
\hline Ex.9 & 1 & 8 & 27 & 64 \\
\hline Ex.10 & 1 & 8 & 27 & 64 \\
\hline \multicolumn{5}{|c|}{ Polygonal plates } \\
\hline Ex.11 & 1 & 8 & 39 & 136 \\
\hline Ex.12 & 1 & 13 & 92 & 430 \\
\hline Ex.13 & 1 & 18 & 198 & 1300 \\
\hline Ex.14 & 1 & 30 & 498 & 4435 \\
\hline \multicolumn{7}{|c|}{ Plates with circular edges } \\
\hline Ex.15 & 1 & 2 & 3 & 4 \\
\hline Ex.16 & 1 & 2 & 3 & 4 \\
\hline Ex.17 & 1 & 4 & 9 & 16 \\
\hline Ex.18 & 1 & 12 & 54 & 160 \\
\hline
\end{tabular}

\section{Numerical Examples and Discussions}

The cyclic polynomials are derived for eighteen plate shapes (Ex.1-18) to calculate number of different combinations in edge conditions. Table 1 presents a list of numbers of different combinations, when one, two, three and four edge conditions are applied along each edge. For typical plate bending problem, there considered three classical conditions, such as free edge (F), simply supported (S) and clamped $(\mathrm{C})$ edges. In this table, the number of conditions 1,2 , and 3 per edge indicates any choices of one edge condition, two conditions and three conditions, respectively, chosen from (F,S,C). A case of four conditions implies, say, including another condition of elastic support, or a little unrealistic sliding edge (slope and shear force both being zero).

As seen (Ex.6) in the table, the maximum number of different combinations are 16,81 and 256 versus Number of conditions $x=2,3$ and 4 at each edge, for Asymmetric trapezoid among examples of "Plates with four straight edges".

Similarly, the maximum number is 8,27 and 64 for (Ex.10) in "Triangular plates", and for most complicated case of "Polygonal plates", it is 30, 498 and 4435 of (Ex.14) (regular) Octagonal plate. In contrast, "Plates with circular edges" provide smaller number of combinations due to no corners (i.e., small number of distinct edges) along curved edges except for (Ex.18).

In previous report [7] dealing with square and rectangular plates only, the number of different combinations was numerically demonstrated to be exact. This was possible by using a software to accommodate any sets of edge conditions for calculating natural frequencies of rectangular plates. Such software, however, is not available to the cases of plates with general shapes discussed in this paper.

An attempt was made next by a trial and error approach to figure out different number of combinations for the two conditions along each edge, and the two edge conditions are represented by $\mathrm{F}$ and $\mathrm{C}$ in the examples.

Figure 17 presents seven different combinations (as listed in Table 1) of a rhombic plate. As expected, in addition to FFFF and CCCC, there is only one case of one $\mathrm{C}$ and three $\mathrm{F}$ (also one $\mathrm{F}$ and three $\mathrm{C}$ ) due to four edges of equal length, while CCFF and CFCF are regarded as different since CCFF cannot be made from CFCF by any rotation and flipping.

The next example is taken from (Ex.5) Symmetric trapezoid, where only symmetry axis exists. There are twelve (as given in the table) possible combinations, as shown in Fig.18. When this symmetry is broken as in (Ex.6) Asymmetric trapezoid, there are no duplication and the number of combinations becomes $2^{4}=16$, which is the same as general quadrilateral plate.

For (Ex.7) Equilateral triangular plate, there are three symmetric axes and the number of combinations is reduced significantly by the symmetry to only four, as shown in Fig.19. Figure 20 presents the combinations of $\mathrm{F}$ and $\mathrm{C}$ for (Ex.8) Equilateral triangle and only one symmetry axis exists. The loss of symmetry from three to one causes the combination being increased to six, as observed in the figure.

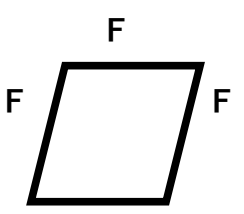

$\mathrm{F}$

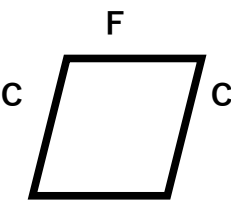

$\mathrm{F}$

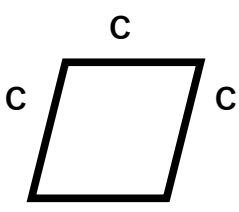

C

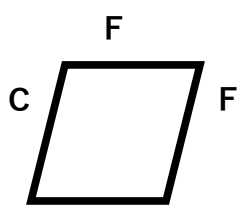

F

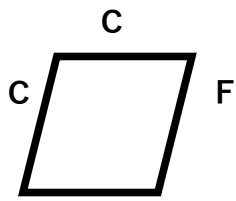

F

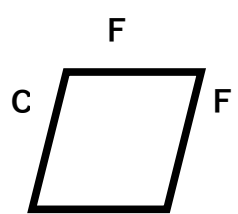

C

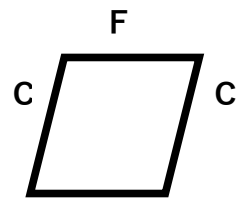

C
Figure 17. Combination of two edge conditions $(\mathrm{F}, \mathrm{C})$ for rhombic plate (Ex.3) 


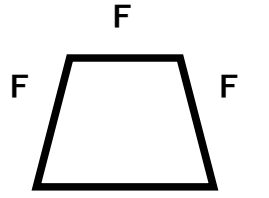

$\mathbf{F}$

C

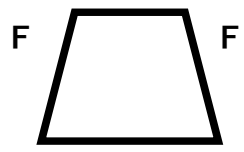

F

C

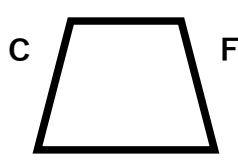

F

C

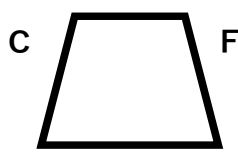

C

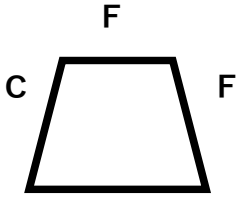

$\mathbf{F}$

F

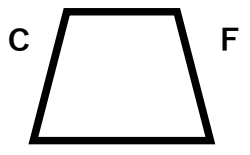

C

C

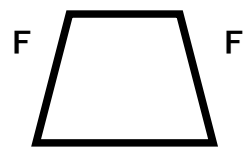

C

$\mathrm{C}$

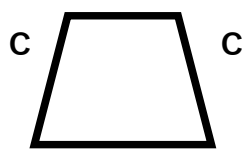

$\mathbf{F}$

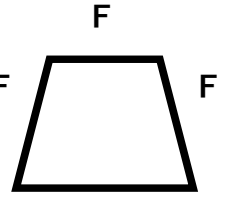

C

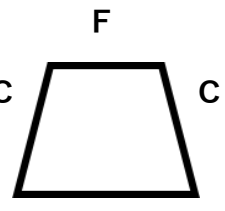

F

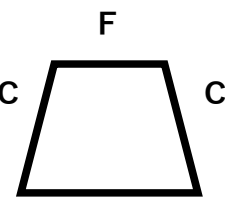

C

$\mathrm{C}$

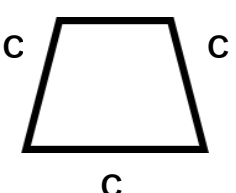

C

Figure 18. Combination of two edge conditions $(F, C)$ for symmetric trapezoidal plate (Ex.5)

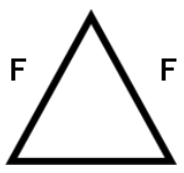

F

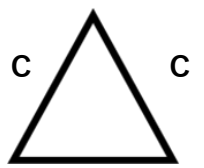

C

Figure 19. Combination of two edge conditions (F, C) for equilateral triangular plate (Ex.7)

Figure 21 presents eight combinations of a regular pentagonal plate. For complicated cases of regular polygonal plates, it is interesting to observe the balance between the increase of edges (increasing the number of combinations) and the increase of symmetry axes (decreasing the number). It is observed that the increase of edges is more dominant than that of symmetry, and in the case of (Ex.14) regular octagonal plates, the number of combinations become significantly large as 30, 498 and 4435 for $x=2,3$ and 4, respectively. For such large number of combinations, it is impossible to figure out all the possible combinations by a trial and error approach.

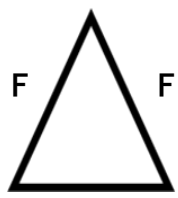

$\mathrm{F}$

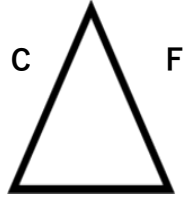

C

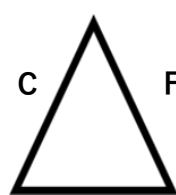

$\mathrm{F}$

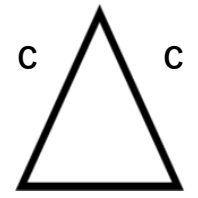

$\mathbf{F}$

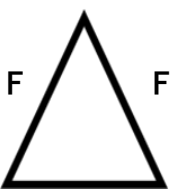

C
Figure 20. Combination of two edge conditions $(\mathrm{F}, \mathrm{C})$ for isosceles triangular plate (Ex.8)

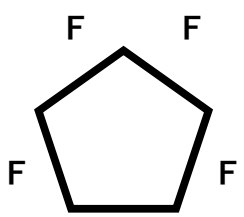

$\mathbf{F}$

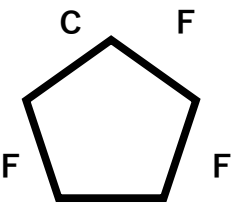

C

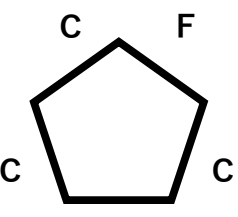

C

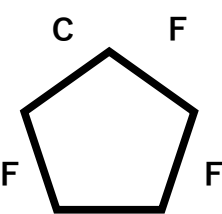

$\mathbf{F}$

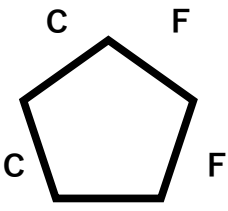

C

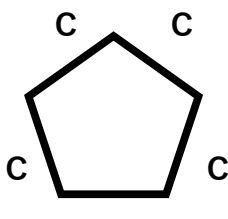

C

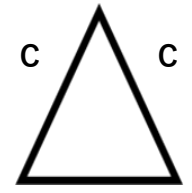

C
Figure 21. Combination of two edge conditions $(F, C)$ for regular pentagonal plate (Ex.11)

\section{Conclusions}

Polya counting theory was developed in the 1930's, and has been applied to a wide range of combinatorial problems in mathematics and chemistry. The present paper extended use of the theory to combination of edge (boundary) conditions that yield different sets of mechanical characteristics, such as stress distribution, deflection, natural frequencies and critical buckling loads, that are found in isotropic flat plates with various geometry.

First, a method for counting was outlined for a case of a rectangular plate. Secondly, eighteen examples of different plate shapes are introduced, and for each of plate shape, a cyclic polynomial was derived. When the number of each edge condition is given for 1,2, 3 and 4, the total number of sets of edge conditions is listed for eighteen examples. Validity of these results is partially established by showing 
actual combinations for rhombic, symmetric trapezoidal, equilateral and isosceles triangular, and regular pentagonal plates in the case of two edge conditions (free edge and clamped edge) applied along each edge. These examples collaborated the validity of the present method. It is hoped that this combinatorics approach will help in summarizing design data books and monographs.

\section{References}

[1] Leissa, A.W., 1993, Vibration of Plates, Acoustical Society of America, (previously, 1969 NASA SP-160, U.S. Government Printing Office, Washington D.C.).
[2] Leissa, A.W. 1973, The free vibration of rectangular plates, J. Sound Vib., vol.31, pp.257-293.

[3] Redfield, J.H., 1927, The theory of group-reduced distributions, American Journal of Mathematics, vol.49, no.3, pp.433-455.

[4] Pólya, G. and Read, R.C., 1987, Combinatorial Enumeration of Groups, Graphs, and Chemical Compounds, Springer-Velag.

[5] Harris, J.M., Hirst, J.L., Mossinghoff, M.J., 2000, Combinatorics and Graph Theory, Springer.

[6] Haigh, C.W., Baker, P.K., 1994, Use of Polya's theorem to enumerate the isomers of seven-coordinate complexes with capped octahedral geometry, Polyhedron, vol. 13, no. 3, pp. 417433.

[7] Narita, Y., 2000, Combinations for the free-vibration behaviors of anisotropic rectangular plates under general edge conditions, Trans. ASME Journal of Applied Mechanics, vol.67, pp.568-573. 